\title{
Estudo clínico da malária falciparum em crianças em Manaus, AM, Brasil
}

\author{
Clinical study of falciparum malaria in children \\ in Manaus, AM, Brazil \\ Elza Noronha, Maria das Graças Costa Alecrim, Gustavo Adolfo \\ Sierra Romero e Vanize Macêdo
}

\begin{abstract}
Resumo As características clínicas da malária falciparum foram estudadas em 61 crianças com idade de 0 a 14 anos, atendidas em centro de referência em Manaus, entre outubro e dezembro de 1997. Os sintomas encontrados foram febre (98,4\%), cefaléia (80,3\%), calafrios $(68,9 \%)$, sudorese $(65,6 \%)$, mialgia (59\%), náuseas $(54,1 \%)$, lombalgia $(49,2 \%)$, vômitos $(49,2 \%)$, tosse $(45,9 \%)$, artralgia $(31,1 \%)$, diarréia $(34,4 \%)$, dispnéia $(8,2 \%)$, convulsões $(8,2 \%)$ e tonturas $(4,9 \%)$. Palidez cutâneo-mucosa e anemia foram observadas mais freqüentemente nas crianças menores de 5 anos. A anemia esteve asssociada aos maiores níveis de parasitemia. Cinqüenta e oito $(91,5 \%)$ pacientes apresentaram malária não complicada, 3 (4,9\%) malária grave e a letalidade foi $1,6 \%$.
\end{abstract}

Palavras-chaves: Malária. Clínica. Plasmodium falciparum. Brasil.

Abstract The clinical characteristics of falciparum malaria were studied among 61 children, aged 0 to 14 treated at a reference center in Manaus, from October to December 1997. The symptoms observed were fever (98.4\%), headache (80.3\%), chills $(68.9 \%)$, perspiration $(65.6 \%)$, myalgia (59.0\%), nausea (54.1\%), lumbar pain (49.2\%), vomiting (49.2\%), cough $(45.9 \%)$, arthralgia (31.1\%), diarrhea (34.4\%), dyspnea (8.2\%), convulsions (8.2\%) and dizziness (4.9\%). Pallor and anaemia were found more frequently in children under five years old. Anaemia was associated with high levels of parasitaemia. Fifty-eight (91.5\%) patients had uncomplicated malaria, $3(4.9 \%)$ had severe malaria and the lethality was $1.6 \%$.

Key-words: Malaria. Clinical. Plasmodium falciparum. Brazil.

A malária falciparum é uma doença tropical com grande importância em saúde pública em virtude das altas taxas de morbidade e mortalidade no mundo, principalmente, se considerarmos a infecção nas crianças, um grupo suscetível às formas graves da doença. Acrescenta-se ainda, o impacto do surgimento e da disseminação de cepas de P. falciparum multirresistentes, fato verificado na Amazônia Brasileira na década de $80^{1}$. A Amazônia legal representa o maior foco malarígeno do país, onde tem sido relatado uma transmissão do tipo hipoendêmica e instável ${ }^{4}$, embora apresente variáveis perfis, de acordo com os agrupamentos populacionais.

Teoricamente, as crianças e os adultos da região apresentam semelhante risco de desenvolver a forma grave da doença. As maiores contribuições para o conhecimento da infecção malárica em crianças referem-se aos trabalhos realizados em áreas de transmissão intensa do $P$. falciparum, como algumas regiões da África, onde a maioria das crianças são

Núcleo de Medicina Tropical e Nutrição da Universidade de Brasília, DF, Instituto de Medicina Tropical do Amazonas, Manaus, AM, Brasil Endereço para correspondência: Dra Elza Noronha. Núcleo de Medicina Tropical e Nutrição, UNB. Caixa Postal 4517, 70919-970 Brasília, DF, Brasil.

Tel: 5561 273-5008/348-2197; fax: $5561273-2811$

e-mail: tropical@.unb.br

Recebido para publicação em 25/5/99. 
infectadas no primeiro ano de vida e é alta a mortalidade infantil ${ }^{67}$. A aquisição de imunidade no hospedeiro humano é um importante condicionante do evento clínico da malária falciparum, sendo influenciada pelo perfil de transmissão e pela idade do indivíduo ${ }^{3}$. Uma tradução clara da influência da idade nas manifestações clínicas da malária é a diferença observada entre adultos e crianças com malária grave. Diferente dos adultos, as crianças, apresentam complicações como malária cerebral, anemia, hipoglicemia e convulsões, entre outros ${ }^{16}$. Entretanto, a imunidade natural adquirida não é o único fator determinante do desfecho clínico da parasitose e deve-se considerar que a compreensão do processo saúde-doença na malária falciparum pressupõe uma visão mais ampla dos fenômenos biológicos conhecidos, pois a maior gravidade da doença depende de interações complexas entre sistema imune, carga genética do hospedeiro, resistência aos antimaláricos, fatores operacionais como o acesso a diagnóstico e tratamento precoces e, possivelmente, fatores de virulência relacionados ao parasita ${ }^{13}$. Portanto, observa-se a necessidade de se estudar a doença em cada contexto geográfico, como nesse trabalho, onde pretendemos avaliar as características clínicas da malária falciparum em crianças procedentes da Amazônia brasileira.

\section{MATERIAL E MÉTODOS}

O estudo foi realizado no Instituto de Medicina Tropical do Amazonas (IMT-AM), centro de referência para doenças infecciosas em Manaus. O número de casos atendidos no serviço corresponde a $58 \%$ do total do município, procedentes da áreas rural e urbana. Cerca de $75 \%$ dos pacientes têm idade acima de 14 anos e predomina a malária por $P$. vivax. A infecção por $P$. falciparum nas crianças representa $20 \%$ dos casos de malária falciparum. Em 1996, a malária falciparum foi a segunda principal causa de internação no serviço correspondendo a 3\% dos casos de malária, não sendo registrados óbitos.

No período de outubro a dezembro de 1997, foram avaliadas todas as crianças de 0 a 14 anos com infecção por $P$. falciparum. Os pacientes com gota espessa positiva eram encaminhados ao ambulatório onde, após a obtenção de consentimento informado dos pais ou responsáveis, procedia-se à anamnese, exame clínico e coleta de sangue para realização da quantificação de leucócitos e plaquetas, dosagem de hematócrito, hemoglobina, glicose, uréia, creatinina, transaminases, bilirrubinas. A pesquisa de plasmódio foi realizada através de punção digital e confecção de gota espessa, com coloração pelo Giemsa e contagem dos parasitas, tendo como referência 100 leucócitos para a estimativa da densidade parasitária ${ }^{2}$. As dosagens hematológicas e bioquímicas foram realizadas por método automatizado, nos aparelhos STK e Ciba Corning Express 550/Plus, sendo usadas as referências do aparelho para valores normais. Em especial, considerou-se anemia quando os valores de hemoglobina estavam abaixo de $11 \mathrm{~g} / \mathrm{dl}$ para as crianças com até cinco anos e $12 \mathrm{~g} / \mathrm{dl}$ para as demais ${ }^{5}$. Os pacientes foram acompanhados com controles clínico e parasitológico nos dias 3, 5, 7, 14, 21, 28 e 35 após o início do tratamento. O seguimento foi feito, preferencialmente, no ambulatório, sendo indicada a internação nos casos graves. Todas as crianças foram avaliadas de acordo com os critérios de gravidade da Organização Mundial de Saúde $(\mathrm{OMS})^{16}$ e tratadas segundo o protocolo do serviço, inclusive para as intercorrências e patologias associadas. Excepcionalmente, foi adotado o ponto de corte de sessenta mil parasitas $/ \mathrm{mm}^{3}$ para o critério de hiperparasitemia, segundo Alecrim 1 . O protocolo de pesquisa foi aprovado pela Comissão de Ética do IMT-AM e cumpriu com as exigências da resolução 196/96 do Conselho Nacional de Saúde sobre pesquisa envolvendo seres humanos. Os dados foram analisados no Programa EPI-INFO versão 6.04. A freqüência dos eventos foi estimada por meio de proporções e seus respectivos Intervalos de Confiança de $95 \%$ com nível de significância de $5 \%$. As proporções foram comparadas pelo teste do Qui-quadrado.

\section{RESULTADOS}

Foram estudadas 61 crianças com malária falciparum, cuja média de idade foi 7,5 anos, a idade mínima observada foi 4 meses e a máxima
14 anos. Não houve diferenças na distribuição por sexo e houve predomínio de mestiços $(91,8 \%)$ e de brancos $(8,2 \%)$. Cerca de oitenta e cinco por 
cento das crianças nasceram no Amazonas. A maioria $(86,3 \%)$ das crianças moravam no município de Manaus e as demais, nos municípios de Careiro da Várzea, Manacapuru, Presidente Figueiredo, Rio Preto da Eva e de Boa Vista, em Roraima. A média de tempo de residência no local foi 3,5 anos (DP $=3,6$ anos). Cerca de $55,7 \%$ moravam em área rural e, 43,8\% em área urbana. Em relação ao local de provável aquisição de infecção, a maioria $(85,2 \%)$ teria sido infectada na área rural, embora tenha ocorrido transmissão urbana $(13,1 \%)$, principalmente nos bairros da periferia de Manaus. Uma (1,6\%) criança adquiriu a doença em área de garimpo. Em relação à exposição prévia, 45,9\% apresentaram episódios anteriores de malária e $31 \%$ havia feito uso de antimaláricos nos últimos 30 dias. Aproximadamente, $19 \%$ das crianças não faziam uso de medida de proteção individual como repelentes ou mosquiteiros.

Os principais sintomas e sinais observados antes do tratamento, bem como a sua duração média estão mostrados na Tabela 1. Não houve

Tabela 1 - Sinais e sintomas em crianças com malária falciparum no IMT-AM-1997 - freqüência e duração.

\begin{tabular}{|c|c|c|c|}
\hline \multirow[t]{2}{*}{ Sintomas/sinais } & \multicolumn{2}{|c|}{ Freqüência } & \multirow[t]{2}{*}{ Duração* } \\
\hline & $\mathrm{n}^{0}$ & $\%$ & \\
\hline Febre & 60 & 98,4 & $5,5(5,7)$ \\
\hline Cefaléia & 49 & 80,3 & $4,1(4,7)$ \\
\hline Calafrios & 42 & 68,9 & $5,3(5,4)$ \\
\hline Sudorese & 40 & 65,6 & $5,4(5,3)$ \\
\hline Mialgia & 36 & 59,0 & $4,5(3,9)$ \\
\hline Náuseas & 33 & 54,1 & $5,2(5,6)$ \\
\hline Lombalgia & 30 & 49,2 & $4,7(3,8)$ \\
\hline Vômitos & 30 & 49,2 & $5,5(5,8)$ \\
\hline Tosse & 28 & 45,9 & $6,2(7,5)$ \\
\hline Diarréia & 21 & 34,4 & $5,7(6,6)$ \\
\hline Artralgia & 19 & 31,1 & $4,9(4,6)$ \\
\hline Dispnéia & 5 & 8,2 & $3,8(1,3)$ \\
\hline Convulsão & 5 & 8,2 & $3,8(6,3)$ \\
\hline Tontura & 3 & 4,9 & $4,0(1,0)$ \\
\hline
\end{tabular}

* Média de dias (desvio padrão).

diferença na sintomatologia entre os pacientes com diferentes níveis de parasitemia, considerando o ponto de corte de cinco mil parasitas $/ \mathrm{mm}^{3}$. Em relação à idade, a lombalgia foi mais freqüente nas crianças de 10 a 14 anos, não sendo possível estabelecer significância estatística entre os demais. Ao exame físico, 95,1\% não apresentavam comprometimento do estado geral. Com relação aos achados anormais, $52,5 \%$ dos pacientes tinham hepatomegalia, $42,6 \%$ esplenomegalia, $37,7 \%$ palidez cutâneo-mucosa e 11,5\% desidratação leve. Apenas uma $(1,6 \%)$ criança era assintomática, porém, estava em acompanhamento para verificação de cura de malária por $P$. vivax e teve a parasitemia inicial calculada em 64 parasitas $/ \mathrm{mm}^{3}$.

As alterações laboratoriais (Tabela 2), mostram que a anemia foi o achado mais importante, sendo verificada em $54,5 \%$ das crianças. Não foi evidenciada anemia grave $(\mathrm{Hb}<5 \mathrm{~g} / \mathrm{dl})$. Houve uma forte associação entre a presença de anemia e os maiores níveis de parasitemia, sendo mais freqüente nas crianças com parasitemia acima de cinco mil parasitas $/ \mathrm{mm}^{3}$ (teste $\mathrm{X}^{2} ; p=0,003$ ). A distribuição de achados clínicos e laboratoriais, entre as faixas etárias, mostrou a correlação estatística significante entre a palidez cutâneo-mucosa e a anemia nas crianças menores de 5 anos (Tabela 3).

Três (4,9\%) crianças (IC 95\% de 1,3 a 14,6) apresentaram malária grave e $58(95,1 \%)$ crianças (IC 95\% de 85,4 a 98,7) malária não complicada. A letalidade foi 1,6\% (IC 95\% de 0,1 a 10), sendo registrado um óbito no período. Em relação aos critérios de gravidade verificados, uma criança de 12 anos apresentou hiperparasitemia, icterícia, insuficiência renal, convulsões e sangramentos espontâneos. Outra de 3 anos, após o tratamento 
Tabela 2- Exames laboratoriais com alterações das 61 crianças com malária falciparum no IMT-AM-1997.

\begin{tabular}{|c|c|c|c|}
\hline \multirow[t]{2}{*}{ Exames laboratoriais } & \multicolumn{2}{|c|}{ Analisados } & \multirow{2}{*}{$\begin{array}{c}\text { Exames com alterações } \\
\%\end{array}$} \\
\hline & $\mathrm{n}^{0}$ & $\%$ & \\
\hline Leucócitos totais & 55 & 90,1 & 18,2 \\
\hline Hematócrito & 55 & 90,1 & 72,7 \\
\hline Hemoglobina & 55 & 90,1 & 54,5 \\
\hline Plaquetas & 54 & 88,5 & 46,3 \\
\hline Glicose & 53 & 86,9 & 2,0 \\
\hline Uréia & 51 & 83,6 & 11,8 \\
\hline Creatinina & 52 & 85,2 & 5,8 \\
\hline AST & 53 & 86,9 & 28,3 \\
\hline ALT & 53 & 86,9 & 26,4 \\
\hline \multicolumn{4}{|l|}{ Bilirrubina } \\
\hline total & 52 & 85,2 & 13,5 \\
\hline indireta & 52 & 85,2 & 9,6 \\
\hline direta & 52 & 85,2 & 34,6 \\
\hline
\end{tabular}

Tabela 3 - Alterações clínicas e de exames laboratoriais nas 61 crianças com malária falciparum, de acordo com a faixa etária, no IMT-AM-1997.

\begin{tabular}{|c|c|c|c|c|c|c|c|}
\hline \multirow{3}{*}{$\begin{array}{l}\text { Alterações clínicas } \\
\text { e laboratoriais }\end{array}$} & \multicolumn{6}{|c|}{ Faixa etária (anos) } & \multirow[b]{3}{*}{$p^{* *}$} \\
\hline & \multicolumn{2}{|c|}{$0-4$} & \multicolumn{2}{|c|}{$5-9$} & \multicolumn{2}{|c|}{$10-14$} & \\
\hline & $\mathrm{n}^{\circ}$ & $\%^{*}$ & $\mathrm{n}^{0}$ & $\%^{*}$ & $\mathrm{n}^{\circ}$ & $\%^{*}$ & \\
\hline Febre & 18 & 33,3 & 17 & 47,1 & 26 & 58,8 & NS \\
\hline Desidratação & 18 & 16,7 & 17 & 5,9 & 26 & 11,5 & NS \\
\hline Palidez & 18 & 61,1 & 17 & 29,4 & 26 & 26,9 & 0,05 \\
\hline Hepatomegalia & 18 & 61,0 & 17 & 47,1 & 26 & 50,0 & NS \\
\hline Esplenomegalia & 18 & 55,6 & 17 & 41,2 & 26 & 34,6 & NS \\
\hline Anemia & 15 & 93,3 & 15 & 53,3 & 25 & 32,0 & 0,001 \\
\hline
\end{tabular}

* percentual de achados anormais ** valor para teste de comparação de proporções entre as faixas etárias.

NS = não significante,.

com mefloquina, evoluiu com hiperparasitemia, infecção pulmonar e febre hemoglobinúrica, e uma terceira, de 11 meses, apresentou queda do estado geral e prostração, no segundo dia de internação, após negativação da parasitemia, apresentou insuficiência respiratória e óbito.

\section{DISCUSSÃO}

O conhecimento do comportamento clínico da malária falciparum em cada contexto epidemiológico é importante para a implementação de medidas ideais de tratamento e de controle. A maioria das crianças avaliadas nesse estudo se infectaram em área rural de Manaus, envolvendo outros municípios numa pequena parcela. Como mostrado no trabalho, a transmissão urbana da malária falciparum em Manaus que já foi descrita anteriormente ${ }^{14}$, ocorreu em menor proporção.

A maioria das crianças apresentaram a forma não complicada da doença, entretanto, a prevalência de malária grave foi, relativamente, alta, considerando o padrão instável de transmissão da malária na região. A prevalência de $4,9 \%$ de doença grave pode ser explicada pelas características da população estudada, pois se trata de uma amostragem de conveniência de casos atendidos em serviço hospitalar com seus possíveis viéses e, provavelmente, pela virulência da cepa envolvida. Um outro aspecto a ser considerado é a importância do tempo de evolução da doença, embora esse estudo não tenha permitido avaliar esse fator prognóstico.

A letalidade foi baixa no grupo estudado, comparada aos dados observados em crianças hospitalizadas em Itaituba no Pará12, embora esteja de acordo com estudos anteriores 
realizados no IMT-AM ${ }^{8}$ e em Rondônia 9 . O óbito verificado foi causado por insuficiência respiratória que, geralmente, está associada a maior mortalidade em crianças africanas ${ }^{10}$.

Em relação aos sintomas, vale ressaltar a ocorrência de convulsões em cinco crianças, embora pareçam estar relacionadas exclusivamente ao $P$. falciparum somente em duas. Nas demais, os fatores determinantes causais relacionaramse com a febre e antecedentes convulsivos. A ocorrência de convulsões tem sido relatada em crianças com malária não complicada e complicada, parecendo haver relação com a infecção pelo $P$. falciparum e idade menor de três anos ${ }^{15}$.

No estudo, ao estabelecermos as relações entre anemia, maiores níveis de parasitemia e baixa idade, levantamos a possibilidade da malária ser um fator importante para explicar os baixos índices de hemoglobina, como é encontrado nas áreas hiperendêmicas ${ }^{1117}$. Entretanto, outras possibilidades devem ser consideradas como os fatores carencial e espoliativo, não avaliados nesse estudo.

Os dados apontam para a importância da anemia nas crianças menores de cinco anos com malária falciparum atendidas no IMT-AM, sugerindo a necessidade da monitorização do nível hemoglobina com o objetivo de diminuir a morbidade pela doença, além das orientações já estabelecidas em relação à importância do acesso rápido ao tratamento adequado na prevenção das formas graves da doença.

\section{AGRADECIMENTOS}

Às crianças e pais pela colaboração, ao Prof. Wilson Duarte Alecrim, pelas facilidades oferecidas para a realização do estudo no IMT-AM e ao Prof. Edgar Merchan-Hamann pelo auxílio na análise de dados.

\section{REFERÊNCIAS BIBLIOGRÁFICAS}

1. Alecrim MGC. Estudo da resistência do P. falciparum às drogas anti-maláricas in vivo e in vitro na Amazônia. Tese de Mestrado, Universidade de Brasília, Brasília, DF,1981.

2. Antuñano FJL. Diagnóstico microscópico de los párasitos de la malaria en la sangre. In: Antuñano FJL, Schmunis G (eds) Diagnóstico de malaria. Organización Panamericana de la Salud, Washington, p.39-50, 1988.

3. Baird JK. Host Age as a Determinant of Naturally Acquired Immunity to Plasmodium falciparum. Parasitology Today 11:105-111,1995.

4. Camargo LMA, Ferreira MU, Krieger H, Camargo EP, Silva LP. Unstable hypoendemic malaria in Rondonia (Western Amazon Region, Brazil): epidemic outbreaks and work - associated incidence in a agroindustrial rural settlement. The American Journal of Tropical Medicine and Hygiene 51:16-25, 1994.

5. Demayer E, Adiels-Tegman M. The prevalence of anemia in the world. World Health Statistics Quartely 38:302-316, 1985.

6. Greenwood BM, Bradley AK, Greenwood AM, Byass P, Jammeh K, Marsh K, Tulloch S, Oldfield FS, Hayes R. Mortality and morbidity from malaria among children in a rural area of the Gambia, West Africa. Transactions of the Royal Society of Tropical Medicine and Hygiene 81: 478-486, 1987.

7. Greenwood BM, Marsh K, Snow EW. Why do some children develop severe malaria? Parasitology Today 7:277-288,1991.

8. Guerra ALPS, Marques GFN, Raposo AA, Dourado S, Alecrim WD. Principais manifestações clínicas e laboratoriais na malária em pediatria no IMTM. In: Resumos do XXXII Congresso da Sociedade Brasileira de Medicina Tropical, Brasília, p.173, 1996.

9. Gutierrez MU. Avaliação da influência dos fatores de proteção sobre a infecção malárica em crianças menores de 12 meses de idade em Costa Marques, Rondônia, Brasil. Tese de Mestrado, Universidade de Brasília, Brasília, DF, 1990.

10. Marsh K, Foster D, Waruiru C, Mwangi I, Winstanley M, Marsh V, Newton C, Winstanley P, Warn P, Peshu N, Pasvol G, Snow R. Indicators of life-threatening malaria in African children. The New England Journal of Medicine 332:1399-1404, 1995.

11. Newton CR, Warn PA, Winstanley PA, Peshu N, Snow RW, Pasvol G, Marsh K. Severe anaemia in children living in a malaria endemic area of Kenia. Tropical Medicine and International Health 2:165-178, 1997.

12. Pinheiro MCN, Crescente JAB, Cardoso BS, Gaspar MG. Malária na infância: um estudo clínico - epidemiológico em área de alta transmissão do Estado do Pará. In: Resumos do XXXII Congresso da Sociedade Brasileira de Medicina Tropical, Brasília, p.176, 1996.

13. Robert F, Ntoumi F, Angel G, Candito D, Rogier C, Fandeur T, Sarthou JL, Mercereau-Puijalon O. Extensive genetic diversity of Plasmodium falciparum isolates collected from patients with severe malaria in Dakar, Senegal. Transactions of Royal Society of Tropical Medicine and Hygiene 90:704-711, 1996. 
14. Suárez-Mutis MC. Estudo do processo de transmissão da malária em uma área de invasão recente na cidade de Manaus - Amazonas. Tese de Mestrado, Instituto Oswaldo Cruz /FIOCRUZ, Rio de Janeiro, RJ, 1997.

15. Wattanagoon Y, Srivillarait S, Looareesuwan S, White NJ. Convulsions in childhood malaria. Transactions of the Royal Society of Tropical Medicine and Hygiene 88:426428,1994 .
16. Warrel DA, Molineaux ME, Beales PF. Severe and complicated malaria. Transactions of the Royal Society of Tropical Medicine and Hygiene 84:1-65, 1990.

17. Weatherall DJ, Abdalla S. The anaemia of Plasmodium falciparum malaria. British Medical Bulletin 38:147-151, 1982. 